\title{
Bigraphical arrangements
}

\author{
Sam Hopkins 1"* and David Perkinson $\rrbracket^{2}$ \\ ${ }^{1}$ Massachusetts Institute of Technology, Cambridge MA, 02139, USA \\ ${ }^{2}$ Reed College, Portland OR, 97202, USA
}

\begin{abstract}
We define the bigraphical arrangement of a graph and show that the Pak-Stanley labels of its regions are the parking functions of a closely related graph, thus proving conjectures of Duval, Klivans, and Martin and of Hopkins and Perkinson. A consequence is a new proof of a bijection between labeled graphs and regions of the Shi arrangement first given by Stanley. We also give bounds on the number of regions of a bigraphical arrangement. The full version of this paper is forthcoming in the Transactions of the American Mathematical Society.

Nous définissons la disposition bigraphical d'un graphe et montrons que les étiquettes Pak-Stanley de ses régions sont les fonctions de stationnement d'un graphe étroitement liés, prouvant ainsi conjectures de Duval, Klivans, et Martin et de Hopkins et Perkinson. Une conséquence est une nouvelle preuve d'une bijection entre les graphes et les régions marquées de l'arrangement Shi premier donné par Stanley. Nous donnons également des limites sur le nombre de régions d'un arrangement bigraphical. La version complète de cet article est à paraître dans les Transactions de l'American Mathematical Society.
\end{abstract}

Keywords: hyperplane arrangements; parking functions; abelian sandpile model

Throughout this extended abstract $G$ will be a simple graph (no multiedges or loops, but not necessarily connected) with vertex set $V=\left\{v_{1}, \ldots, v_{n}\right\}$ and edge set $E$. A (real) hyperplane arrangement is a finite collection of affine hyperplanes in Euclidean space. Our object of study is the bigraphical arrangement of $G$, so called because it associates two hyperplanes to each edge of the graph.

Definition 0.1. For each edge $\left\{v_{i}, v_{j}\right\} \in E$, choose real numbers $a_{i j}$ and $a_{j i}$ such that there exists $x \in \mathbb{R}^{n}$ with $x_{i}-x_{j}<a_{i j}$ and $x_{j}-x_{i}<a_{j i}$ for all $\left\{v_{i}, v_{j}\right\} \in E$. We call these numbers parameters and we call $A:=\left\{a_{i j}\right\}$ a parameter list. The bigraphical arrangement $\Sigma_{G}(A)$ is the set of $2|E|$ hyperplanes,

$$
\Sigma_{G}(A):=\left\{x_{i}-x_{j}=a_{i j}:\left\{v_{i}, v_{j}\right\} \in E\right\}
$$

The regions of $\Sigma_{G}(A)$ are the connected components of $\mathbb{R}^{n} \backslash \Sigma_{G}(A)$. The central region is the region defined by $x_{i}-x_{j}<a_{i j}$ for all $\left\{v_{i}, v_{j}\right\} \in E$. The above condition on $A$ guarantees that the central region is nonempty.

\footnotetext{
${ }^{*}$ Email: shopkinsemit.edu

$\dagger$ Email: davidp@reed.edu

1365-8050 @ 2014 Discrete Mathematics and Theoretical Computer Science (DMTCS), Nancy, France
} 
Several authors have connected hyperplane arrangements to graphs in various ways. The graphical arrangement (Stanley, 2007, p. 414) of $G$,

$$
\left\{x_{i}-x_{j}=0:\left\{v_{i}, v_{j}\right\} \in E \text { with } i<j\right\},
$$

associates a single hyperplane to each edge of $G$. One interesting property of the graphical arrangement is that its characteristic polynomial is the chromatic polynomial of $G$. Thus, the graphical arrangement encodes information about colorings of $G$. As we shall see, the bigraphical arrangement encodes information about the parking functions of $G_{\text {. }}$, the graph obtained from $G$ by adding a special sink vertex $v_{0}$ and an edge between $v_{0}$ and each vertex $v \in V$. For background on parking functions, see $\S 2$.

Shi (1986), in his study of the Kazhdan-Lusztig cells of affine Weyl groups of type $\tilde{A}_{n-1}$, introduced the Shi arrangement,

$$
\left\{x_{i}-x_{j}=0,1: 1 \leq i<j \leq n\right\} .
$$

He proved that the number of regions of this arrangement is $(n+1)^{n-1}$, Cayley's formula for the number of spanning trees of the complete graph $K_{n+1}$. Stanley (1996), in collaboration with Pak, was the first to give a bijective proof of this result by labeling the regions of the Shi arrangement with parking functions. (There are several well-known bijections between parking functions and spanning trees.) Stanley and Pak's procedure labels the central region of the Shi arrangement with the parking function $00 \ldots 0$. It then inductively labels the other regions by moving outwards and increasing the $i$ th coordinate of a region's label whenever a hyperplane is crossed that corresponds to an increase in $x_{i}$. We call the resulting labels the Pak-Stanley labels of the regions of an arrangement. Duval et al. (2011) defined the G-Shi arrangement,

$$
\left\{x_{i}-x_{j}=0,1:\left\{v_{i}, v_{j}\right\} \in E \text { with } i<j\right\},
$$

and conjectured that the Pak-Stanley labels of the $G$-Shi arrangement are the parking functions of $G$. We prove this conjecture as a consequence of Corollary 2.8. The $G$-Shi arrangement is in fact a special kind of bigraphical arrangement.

In Hopkins and Perkinson (2012a), the authors studied the G-semiorder arrangement,

$$
\left\{x_{i}-x_{j}=1:\left\{v_{i}, v_{j}\right\} \in E\right\},
$$

another special kind of bigraphical arrangement. They showed that the Pak-Stanley labels of the $G$ semiorder are the $G$.-parking functions sought by Duval, Klivans, and Martin. It was also conjectured in Hopkins and Perkinson (2012a) that if one were to slide the hyperplanes of the $G$-semiorder arrangement along their normals, although some regions are destroyed and others are created, so long as the central region is preserved the set of parking function labels remains the same. In this way, one could deform the $G$-semiorder arrangement into the $G$-Shi arrangement and show that the $G$-Shi arrangement has the expected set of labels. Figure 1 depicts this sliding procedure when $G=K_{3}$.

Our Corollary 2.8 establishes this conjecture: the Pak-Stanley labels of any $\Sigma_{G}(A)$ are the parking functions of $G_{\text {. }}$ (Remark 2.9 indicates how, in addition, all the parking functions of $G$ with respect to each of its vertices are encoded in the regions of $\Sigma_{G}(A)$.) Although our research was motivated by the sliding conjecture, we do not in fact use any notion of "sliding" hyperplanes here and our proof does not rely on the results of the previous paper Hopkins and Perkinson (2012a). Rather, we prove from scratch that for any bigraphical arrangement the Pak-Stanley labels are as claimed by moving outwards from the central region and inductively verifying that each parking function appears as a label. In proving 
Corollary 2.8, we generalize a result of Benson et al. (2010), who show that acyclic total orientations of $G$ correspond to maximal parking functions of $G$. We show that certain families of partial orientations of $G$ defined in $\$ 1$ correspond to all of the parking functions of $G$. If $G$ is the complete graph $K_{n}$ and the parameter list $A$ corresponds to the Shi arrangement, Corollary 2.8 provides an alternate proof of the bijection of Pak and Stanley between regions of the Shi arrangement and parking functions.

Example 0.2. The following are examples of bigraphical arrangements:

1. Setting $a_{i j}=1$ for all $i, j$ gives the $G$-semiorder arrangement, studied in Hopkins and Perkinson (2012a). We will use SEMI to denote the parameter list of the $G$-semiorder arrangement and thus denote the $G$-semiorder arrangement by $\Sigma_{G}(\mathrm{SEMI})$.

2. Setting $a_{i j}=1$ if $i<j$ and 0 otherwise gives the $G$-Shi arrangement, the subject of a conjecture in Duval et al. (2011) that we establish as a consequence of Corollary 2.8. We will use SHI to denote the parameter list of the $G$-Shi arrangement and thus denote the $G$-Shi arrangement by $\Sigma_{G}(\mathrm{SHI})$.

3. Let $\eta=\left(\ell_{1}, \ldots, \ell_{n}\right) \in \mathbb{Z}_{>0}^{n}$. Setting $a_{i j}=\ell_{i}$ for all $i, j$ gives what we call the $(G, \eta)$-interval order arrangement.

Taking $G$ to be the complete graph $K_{n}$ recovers the normal semiorder, Shi, and interval order arrangements. See Stanley (2007) for definitions of these arrangements, as well as for basic concepts from the theory of hyperplane arrangements, in particular, that of the characteristic polynomial.

From now on we assume we have fixed some parameter list $A$. Note that $\Sigma_{G}(A)$ having a nonempty central region is essentially equivalent to $A$ having only positive entries. If the $a_{i j}$ are all positive, then the origin satisfies $x_{i}-x_{j}<a_{i j}$ for all $\left\{v_{i}, v_{j}\right\} \in E$. On the other hand, suppose $\Sigma_{G}(A)$ has a nonempty central region and that $p$ is a point in this region. Then the translation $x \mapsto x-p$ maps $\Sigma_{G}(A)$ to a bigraphical arrangement whose parameter list has positive entries.

In \$1. we develop a correspondence between regions of $\Sigma_{G}(A)$ and partial orientations of $G$. In $\$ 2$, we prove our main result, Corollary 2.8, which says that the Pak-Stanley labeling of any $\Sigma_{G}(A)$ yields the set of parking functions of $G_{\text {. }}$ In $\$ 3$, we bound the number of regions of $\Sigma_{G}(A)$ for arbitrary $A$ and we find its characteristic polynomial when $A$ is generic. The characteristic polynomial of a generic $\Sigma_{G}(A)$ turns out to be related to the Tutte polynomial of $G$.

For the proofs which have been omitted from this extended abstract, see the full version of this paper which is Hopkins and Perkinson (2012b).

\section{Orientations and the regions of a bigraphical arrangement}

Definition 1.1. A partial orientation of $G$ is a choice of directions for a subset of the edges of $G$. Formally, a step is an ordered pair $(u, v) \in V \times V$ such that $\{u, v\} \in E$, and a partial orientation $\mathcal{O}$ is a set of steps with the property that if $(u, v) \in \mathcal{O}$, then $(v, u) \notin \mathcal{O}$. We say $\mathcal{O}$ is acyclic if it does not contain a cycle of steps.

Definition 1.2. Let $\mathcal{O}$ be a partial orientation. If $e=\{u, v\} \in E$ and $(u, v) \in \mathcal{O}$, then despite the ambiguity, we write $e \in \mathcal{O}$ and say $e$ is oriented. In that case, we think of $e$ as an arrow from $u$ to $v$ and write $e^{-}=u$ and $e^{+}=v$. If neither $(u, v)$ nor $(v, u)$ is in $\mathcal{O}$, we write $e \notin \mathcal{O}$ and say that $e$ is an unoriented or blank edge. The indegree of $u \in V$ relative to $\mathcal{O}$, denoted $\operatorname{indeg}_{\mathcal{O}}(u)$, is the number of 

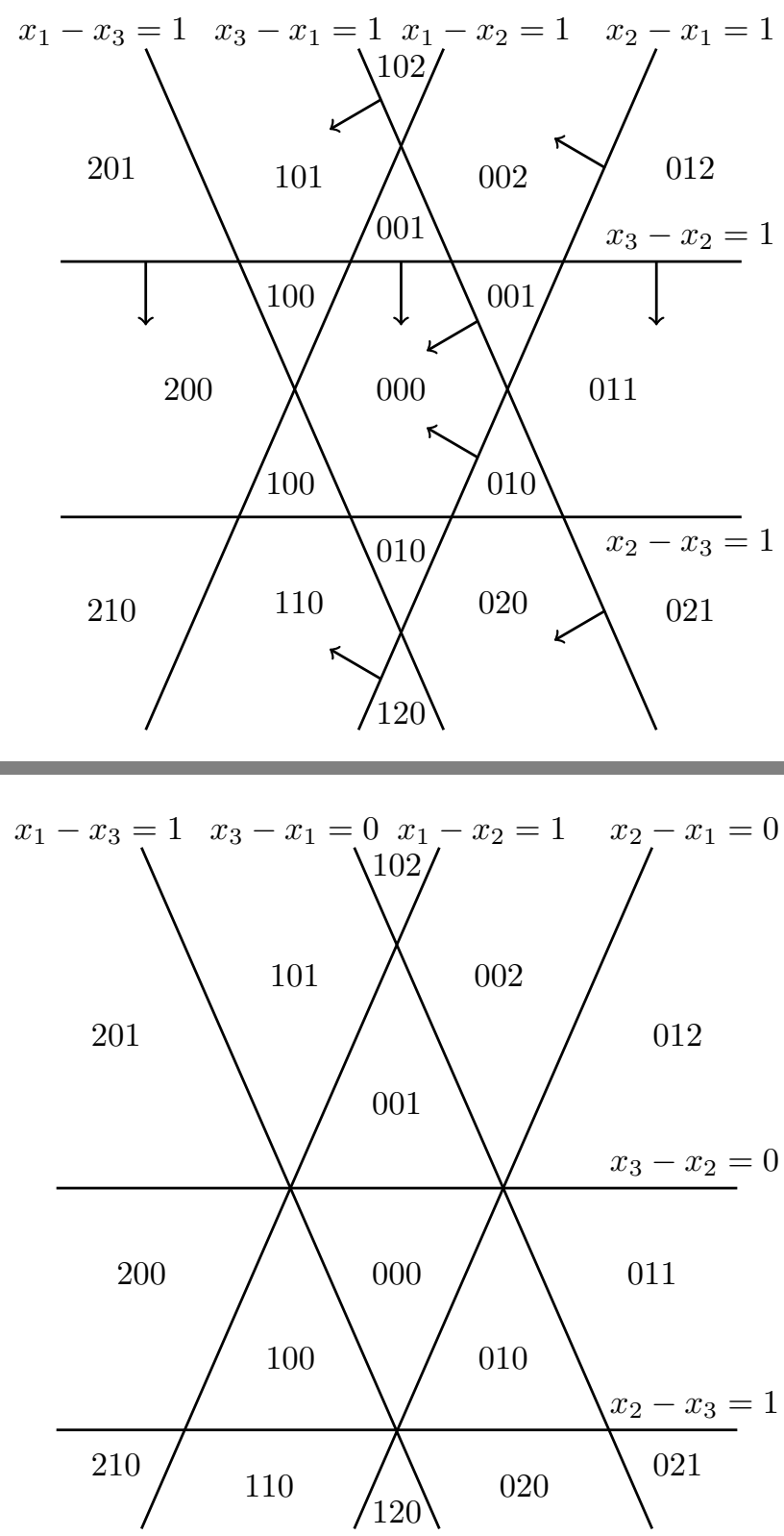

Fig. 1: Hyperplanes in the $K_{3}$-semiorder arrangement (above) are slid in the directions indicated by the arrows to yield the $K_{3}$-Shi arrangement (below). The set of Pak-Stanley labels, strings inside each region, remains the same. 
edges $e \in \mathcal{O}$ such that $e^{+}=u$. Similarly, the outdegree of the vertex $u \in V$ relative to $\mathcal{O}$ is the number of edges $e \in \mathcal{O}$ such that $e^{-}=u$. The degree of $u$ is the number of $e \in E$ containing $u$.

Notation. Partial orientations naturally serve as labels for the regions of bigraphical arrangements. Suppose $R$ is a region of $\Sigma_{G}(A)$. Define $\mathcal{O}_{R}$ to be the partial orientation obtained by (i) $\left(v_{i}, v_{j}\right) \in \mathcal{O}_{R}$ if $\left\{v_{i}, v_{j}\right\} \in E$ and $x_{j}>x_{i}+a_{j i}$ in $R$, and (ii) all other edges are blank. We now classify exactly which partial orientations are labels of regions of $\Sigma_{G}(A)$.

Definition 1.3. Let $\mathcal{O}$ be a partial orientation. A step $(u, v)$ is compatible with $\mathcal{O}$ if $(v, u) \notin \mathcal{O}$. In other words, a step $e$ is compatible with $\mathcal{O}$ if $e \in \mathcal{O}$ or $e$ is a blank edge of $\mathcal{O}$. A potential cycle for $\mathcal{O}$ is a set $C=\left\{\left(u_{1}, u_{2}\right),\left(u_{2}, u_{3}\right), \ldots,\left(u_{k}, u_{1}\right)\right\}$ of steps compatible with $\mathcal{O}$.

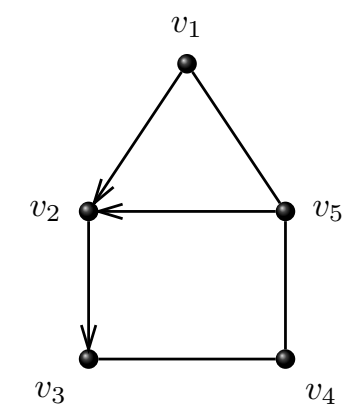

Fig. 2: A partial orientation $\mathcal{O}$. Consider the potential cycle $C:=\left\{\left(v_{5}, v_{2}\right),\left(v_{2}, v_{3}\right),\left(v_{3}, v_{4}\right),\left(v_{4}, v_{5}\right)\right\}$ for $\mathcal{O}$. We have $\nu_{\text {SEMI }}(C, \mathcal{O})=0$, so $\mathcal{O}$ is not SEMI-admissible. However, note that $\mathcal{O}$ is $\mathrm{SHI}$-admissible.

Definition 1.4. Let $\mathcal{O}$ be a partial orientation. The score of a step $e=\left(v_{i}, v_{j}\right)$ compatible with $\mathcal{O}$ is

$$
\nu_{A}(e, \mathcal{O})=\left\{\begin{aligned}
a_{i j} & \text { if }\left\{v_{i}, v_{j}\right\} \notin \mathcal{O}, \\
-a_{j i} & \text { if }\left(v_{i}, v_{j}\right) \in \mathcal{O} .
\end{aligned}\right.
$$

The score of a potential cycle $C$ for $\mathcal{O}$ is

$$
\nu_{A}(C, \mathcal{O})=\sum_{e \in C} \nu(e, \mathcal{O})
$$

When the parameter list is clear from context, we omit the subscript and we write $\nu(C, \mathcal{O}):=\nu_{A}(C, \mathcal{O})$.

Definition 1.5. Let $\mathcal{O}$ be a partial orientation. A potential cycle for $\mathcal{O}$ is $b a d$ if it has a nonpositive score. We say $\mathcal{O}$ is $A$-admissible if it has no bad potential cycles.

Figure 2 gives an example of a partial orientation that is SHI-admissible but not SEMI-admissible.

Theorem 1.6. The regions of $\Sigma_{G}(A)$ are in bijection with the A-admissible partial orientations of $G$. The bijection is given by $R \mapsto \mathcal{O}_{R}$.

Proof. The proof relies on Farkas' lemma. 
Notation. From now on, for an $A$-admissible partial orientation $\mathcal{O}$, we will use $\operatorname{rg}(\mathcal{O})$ to denote the unique region of $\Sigma_{G}(A)$ satisfying $\mathcal{O}=\mathcal{O}_{\operatorname{rg}(\mathcal{O})}$.

Definition 1.7. Let $\mathcal{A}$ be a hyperplane arrangement in $\mathbb{R}^{n}$ and let $W$ be the subspace of $\mathbb{R}^{n}$ spanned by the normals of the hyperplanes in $\mathcal{A}$. We say that a region $R$ of $\mathcal{A}$ is relatively bounded if $R \cap W$ is bounded. The essentialization of $\mathcal{A}$ is $\mathcal{A} \cap W$ considered as a hyperplane arrangement in $W \simeq \mathbb{R}^{k}$, where we have $k=\operatorname{dim}(W)$.

Theorem 1.8. The relatively bounded regions of $\Sigma_{G}(A)$ are in bijection with the A-admissible partial orientations $\mathcal{O}$ of $G$ for which every step in $\mathcal{O}$ belongs to some potential cycle. The bijection is again given by $R \mapsto \mathcal{O}_{R}$.

Proof. Again we use Farkas' lemma.

\section{Parking functions and the regions of a bigraphical arrangement}

In this section, we explain how the indegree sequences of partial orientations of $G$ are closely related to the parking functions of $G_{\bullet}$, the graph obtained from $G$ by adding a vertex $v_{0}$ and an edge between $v_{0}$ and each $v \in V$. We will use $V_{\text {. }}$ and $E$. to denote the vertex and edge set, respectively, of $G_{\text {. . Our goal is to }}$ show that a natural set of labels for the regions of $\Sigma_{G}(A)$ are the set of parking functions of $G$. Let $\mathbb{Z V}$ denote the free abelian group on the vertices in $V$.

Definition 2.1. Let $\mathcal{O}$ be a partial orientation of $G$. The indegree sequence of $\mathcal{O}$, denoted indeg $(\mathcal{O})$, is $\sum_{i=1}^{n} \operatorname{indeg}_{\mathcal{O}}\left(v_{i}\right) v_{i} \in \mathbb{Z} V$.

Definition 2.2. A parking function $c=\sum_{i=1}^{n} c_{i} v_{i}$ of $G$. with respect to $v_{0}$ is an element of $\mathbb{Z} V$ such that for every non-empty subset $W \subseteq V$, there exists $v_{i} \in W$ with $0 \leq c_{i}<d_{W}\left(v_{i}\right)$, where $d_{W}\left(v_{i}\right)$ is the number of edges $e=\left\{v_{i}, u\right\} \in E_{\text {. with }} u \in V$. $\backslash W$. For $c, c^{\prime} \in \mathbb{Z} V$, we write $c \leq c^{\prime}$ if $c_{i} \leq c_{i}^{\prime}$ for all $0 \leq i \leq n$. A parking function $c^{\prime}$ is maximal if $c^{\prime} \leq c$ for any parking function $c$ implies $c=c^{\prime}$.

Graphical parking functions were first formally introduced in Postnikov and Shapiro (2004). However, the essentially equivalent notion of superstable configurations has been studied for longer in the context of the abelian sandpile model; see (Hopkins and Perkinson, 2012a, \$2.4) for a definition of these and their connection to parking functions. One easy observation from the above definition is that if $c^{\prime}$ is a maximal parking function and $c \in \mathbb{Z V}$ with $0 \leq c \leq c^{\prime}$, then $c$ is a parking function as well. The following characterization of maximal parking functions is Theorem 3.1 of Benson et al. (2010):

Theorem 2.3. A total orientation of a graph is a partial orientation where every edge is oriented. A source of a total orientation is a vertex whose outdegree equals its degree. The acyclic total orientations of $G$. with unique source $v_{0}$ are in bijection with the maximal parking functions of $G$. with respect to $v_{0}$. The bijection is given by $\mathcal{O} \mapsto \operatorname{indeg}(\mathcal{O})-\sum_{i=1}^{n} v_{i}$.

Proposition 2.4. The set

$$
\{\operatorname{indeg}(\mathcal{O}): \mathcal{O} \text { is an acyclic partial orientation of } G\}
$$

is the set of parking functions of $G$. with respect to $v_{0}$.

Theorem 1.6 makes clear that an $A$-admissible partial orientation is necessarily acyclic. What remains to be proven is that any parking function of $G$. can be realized as $\operatorname{indeg}(\mathcal{O})$ for some $A$-admissible $\mathcal{O}$. We 
are therefore interested in building $A$-admissible partial orientations with particular indegree sequences. The following "topological" lemma will allow us to build up $A$-admissible partial orientations from other $A$-admissible partial orientations with some control over the resulting indegrees.

Lemma 2.5. Let $\mathcal{O}$ be an $A$-admissible partial orientation, and let $W \subseteq V$ be a subset of the vertices of $G$ satisfying:

1. there do not exist $u \in W^{c}, w \in W$ with $(w, u) \in \mathcal{O}$;

2. there is some $u \in W^{c}, w \in W$ such that $\{u, w\}$ is a blank edge of $\mathcal{O}$.

Then there exists $u \in W^{c}$ and $w \in W$ such that $\mathcal{O} \cup\{(u, w)\}$ is also A-admissible.

Proof. Omitted.

Theorem 2.6. Let $\mathcal{O}$ be an acyclic partial orientation of $G$. Then there exists an A-admissible partial orientation $\mathcal{O}^{\prime}$ such that $\operatorname{indeg}(\mathcal{O})=\operatorname{indeg}\left(\mathcal{O}^{\prime}\right)$.

Proof. The proof is a recursive application of the previous lemma.

We are now prepared to prove the main result of this section, Corollary 2.8, which establishes a conjecture of Hopkins and Perkinson (2012a). Indeed, Corollary 2.8 subsumes the main result of that paper (which was proved in a different way using the abelian sandpile model).

Definition 2.7. The following procedure is called the Pak-Stanley labeling of a bigraphical arrangement. It labels each region with an element of $\mathbb{Z} V$. Label the central region of $\Sigma_{G}(A)$ with 0 . Put the central region in a queue, $Q$. Then, as long as $Q$ is not empty:

1. Remove the first region $R$ from $Q$.

2. For each unlabeled region $R^{\prime}$ bordering $R$ :

(a) Determine the unique indices $i \neq j$ such that $x_{j}-x_{i}<a_{j i}$ in $R$ but $x_{j}>x_{i}+a_{j i}$ in $R^{\prime}$.

(b) If $R$ is labeled by $c=\sum_{k=1}^{n} c_{k} v_{k}$, then label $R^{\prime}$ by $c^{\prime}=c+v_{j}$.

(c) Add $R^{\prime}$ to the end of $Q$.

Let $\lambda(R)$ denote the Pak-Stanley label of the region $R$.

Corollary 2.8. The set

$$
\left\{\lambda(R): R \text { is a region of } \Sigma_{G}(A)\right\}
$$

is the set of all parking functions of $G$. with respect to $v_{0}$.

Proof. It is easy to verify inductively that $\lambda(R)=\operatorname{indeg}\left(\mathcal{O}_{R}\right)$. By Proposition 2.4, for any parking function $c$ of $G_{\bullet}$, there is some acyclic partial orientation $\mathcal{O}$ such that indeg $(\mathcal{O})=c$. Finally, by Theorem 2.6, we can find an $A$-admissible orientation $\mathcal{O}^{\prime}$ with the same indegree sequence as $\mathcal{O}$, so we have $\lambda\left(\operatorname{rg}\left(\mathcal{O}^{\prime}\right)\right)=c$. 
Remark 2.9. In (Hopkins and Perkinson, 2012a, §4), it is shown that

$$
\begin{aligned}
\{c \in \mathbb{Z} V: c & =\operatorname{indeg}(\mathcal{O})-\sum_{i=1}^{n} v_{i} \text { for some acyclic partial orientation } \mathcal{O} \text { of } G, \\
c_{i} & \left.=-1, \text { and } c_{j} \geq 0 \text { for all } j \neq i\right\}
\end{aligned}
$$

is the set of parking functions of $G$ with respect to $v_{i}$. Thus, in light of Theorem 1.6. Proposition 2.4 and Theorem 2.6 the parking functions of $G$ with respect to each of its vertices are encoded in the regions of $\Sigma_{G}(A)$.

Corollary 2.10. The number of regions of $\Sigma_{G}(A)$ is at least the number of spanning trees of $G$.

\section{Number of regions of a bigraphical arrangement}

We have already seen that the number of regions of $\Sigma_{G}(A)$ is at least the number of spanning trees of $G_{\text {. }}$. In this section we give further bounds on the number of regions of $\Sigma_{G}(A)$. The graph $G$ remains fixed, but we will allow the parameter list $A$ to vary (while always maintaining a central region).

Notation. We will denote the number of regions of $\Sigma_{G}(A)$ by $r\left(\Sigma_{G}(A)\right)$ and the number of relatively bounded regions by $b\left(\Sigma_{G}(A)\right)$.

Definition 3.1. Let $\mathcal{A}$ be a hyperplane arrangement. The hyperplanes $H_{1}, \ldots, H_{k}$ in $\mathcal{A}$ are linearly independent if their normals are linearly independent. The arrangement $\mathcal{A}$ is generic if

$$
H_{1} \cap \cdots \cap H_{k} \neq \emptyset \quad \Leftrightarrow \quad H_{1}, \ldots, H_{k} \text { are linearly independent }
$$

for all subsets $\left\{H_{1}, \ldots, H_{k}\right\} \subseteq \mathcal{A}$.

For instance, $\Sigma_{G}(A)$ is generic when the $a_{i j}$ are algebraically independent. We will use GEN to denote the parameter list of an arbitrary generic bigraphical arrangement.

Theorem 3.2. For any generic bigraphical arrangement, the characteristic polynomial of $\Sigma_{G}(\mathrm{GEN})$ is given by

$$
\chi_{\Sigma_{G}(\mathrm{GEN})}(t)=(-2)^{n-1} t T_{G}(1-t / 2,1),
$$

where $T_{G}(x, y)$ is the Tutte polynomial of $G$. Consequently,

$$
\begin{aligned}
& r\left(\Sigma_{G}(\mathrm{GEN})\right)=2^{n-1} T_{G}(3 / 2,1) ; \\
& b\left(\Sigma_{G}(\mathrm{GEN})\right)=2^{n-1} T_{G}(1 / 2,1) .
\end{aligned}
$$

Proof. See (Stanley, 2007, p. 412).

Definition 3.3. Let $\mathcal{O}$ be a partial orientation. A potential cycle for $\mathcal{O}$ is very bad if has a negative score. We say $\mathcal{O}$ is almost-A-admissible if it is not $A$-admissible but it has no very bad potential cycles. We say that $\mathcal{O}$ is far-from-A-admissible if it has a very bad potential cycle. 
Proposition 3.4. For an almost-A-admissible partial orientation $\mathcal{O}$, define $w(\mathcal{O})$ to be the number of steps e of $G$, including blanks of $\mathcal{O}$, belonging to some potential cycle $C$ for $\mathcal{O}$ with $\nu_{A}(C, \mathcal{O})=0$. Define $z(\mathcal{O})$ to be the maximum $k$ such that there exists disjoint potential cycles $C_{1}, \ldots, C_{k}$ for $\mathcal{O}$ with $\nu_{A}\left(C_{i}, \mathcal{O}\right)=0$ for all $i$. Then

$$
\sum_{\mathcal{O}} \frac{1}{2^{w(\mathcal{O})}} \leq r\left(\Sigma_{G}(\mathrm{GEN})\right)-r\left(\Sigma_{G}(A)\right) \leq \sum_{\mathcal{O}} \frac{1}{2^{z(\mathcal{O})}}
$$

where the sum is over all almost-A-admissible partial orientations $\mathcal{O}$.

Proof. Omitted.

Example 3.5. Consider the cycle graph $C_{n}$, labeled as below:

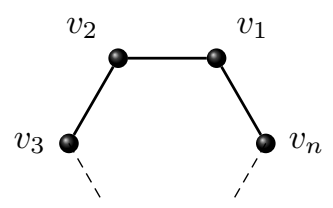

The right inequality from Proposition 3.4 becomes an equality for $C_{n}$ : for any almost- $A$-admissible $\mathcal{O}$, there is only one potential cycle $C$ for $\mathcal{O}$ for which we have $\nu_{A}(C, \mathcal{O})=0$. In fact we have,

$$
\begin{aligned}
& r\left(\Sigma_{C_{n}}(A)\right)=r\left(\Sigma_{C_{n}}(\mathrm{GEN})\right)-\mid\{\mathcal{O}: \mathcal{O} \text { is almost- } A \text {-admissible }\} \mid / 2 ; \\
& b\left(\Sigma_{C_{n}}(A)\right)=b\left(\Sigma_{C_{n}}(\mathrm{GEN})\right)-\mid\{\mathcal{O}: \mathcal{O} \text { is almost- } A \text {-admissible }\} \mid / 2 .
\end{aligned}
$$

This lets us compute exact formulas for $r\left(\Sigma_{C_{n}}(A)\right)$. For instance, one can show

$$
\begin{aligned}
r\left(\Sigma_{C_{n}}(\mathrm{SEMI})\right) & = \begin{cases}3^{n}-2^{n} & \text { if } n \text { is odd, } \\
3^{n}-2^{n}-\left(\begin{array}{c}
n \\
n / 2
\end{array}\right) & \text { if } n \text { is even. }\end{cases} \\
b\left(\Sigma_{C_{n}}(\mathrm{SEMI})\right) & = \begin{cases}2^{n}-1 & \text { if } n \text { is odd }, \\
2^{n}-1-\left(\begin{array}{c}
n \\
n / 2
\end{array}\right) & \text { if } n \text { is even. }\end{cases} \\
r\left(\Sigma_{C_{n}}(\mathrm{SHI})\right) & =3^{n}-2^{n}-n . \\
b\left(\Sigma_{C_{n}}(\mathrm{SHI})\right) & =2^{n}-1-n .
\end{aligned}
$$

We omit the verification of these formulae.

Corollary 3.6. The maximum number of regions of $\Sigma_{G}(A)$ over all parameter matrices $A$ is equal to $2^{n-1} T_{G}(3 / 2,1)$. This maximum is achieved by $\Sigma_{G}(\mathrm{GEN})$.

Proof. This follows from Theorem 3.2 and Proposition 3.4 .

In fact, the converse of Corollary 3.6 is true as well: any parameter list $A$ that maximizes the number of regions of $\Sigma_{G}(A)$ is generic. Thus the problem of maximizing the number of regions of a bigraphical arrangement is completely understood. On the other hand, minimizing the number of regions is much more delicate. We can slightly refine the lower bound for the number of regions given by Corollary 2.10 by considering the degrees of the parking functions of $G_{\text {. }}$. 
Definition 3.7. The degree of a parking function $c=\sum_{i=1}^{n} c_{i} v_{i}$ of $G$. with respect to $v_{0}$ is given by $\operatorname{deg}(c):=\sum_{i=1}^{n} c_{i}$.

Proposition 3.8. Let $g:=\left|E_{\bullet}\right|-\left|V_{\bullet}\right|+1=|E|$ be the genus of $G_{\text {. }}$ For $0 \leq i \leq g$, define:

$$
\begin{aligned}
h_{i}\left(G_{\bullet}\right) & :=\mid\left\{c: c \text { is a parking function of } G . \text { with respect to } v_{0} \text { and } \operatorname{deg}(c)=i\right\} \mid . \\
p_{i}(G, A) & :=\mid\{\mathcal{O}: \mathcal{O} \text { is an A-admissible partial orientation of } G \text { and }|\mathcal{O}|=i\} \mid .
\end{aligned}
$$

Then $h_{i}\left(G_{\bullet}\right) \leq p_{i}(G, A)$ for all $i$.

The lower bound from Corollary 2.10 is sometimes sharp, as in the case of the complete graph $K_{n}$.

Proposition 3.9. The minimum number of regions of $\Sigma_{K_{n}}(A)$ over all parameter matrices $A$ is given by $(n+1)^{(n-1)}$. This minimum is achieved by $\Sigma_{K_{n}}(\mathrm{SHI})$.

Proof. Shi (1986) proved that the number of regions of the Shi arrangement is given by $(n+1)^{(n-1)}$, the number of spanning trees of $K_{n+1}$.

Remark 3.10. Our Corollary 2.8 provides an alternative proof of the bijection of Stanley $(1996)$ between regions of the Shi arrangement and parking functions.

However, the lower bound from Corollary 2.10 is in general not sharp:

Example 3.11. Consider the path graph $P_{3}$, labeled as below:

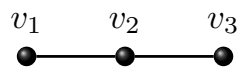

Then the essentialization of any $\Sigma_{P_{3}}(A)$ looks like

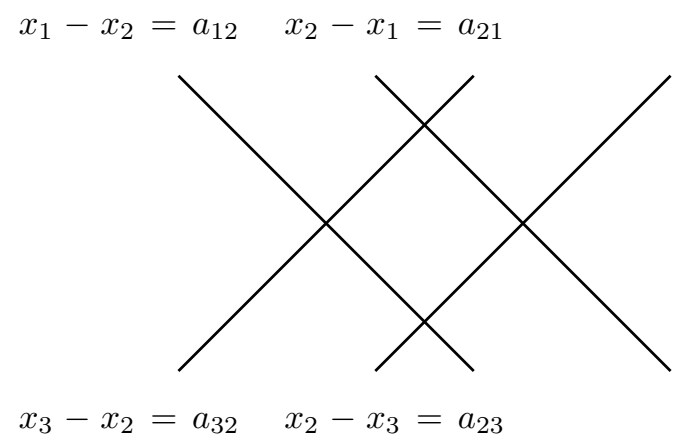

So $r\left(\Sigma_{P_{3}}(A)\right)=9$ for any $A$, but there are only 8 parking functions of $P_{3}$. with respect to $v_{0}$ :

$$
0, v_{1}, v_{2}, v_{3}, v_{1}+v_{2}, v_{1}+v_{3}, 2 v_{2}, v_{2}+v_{3}
$$

Also, $\Sigma_{G}(\mathrm{SHI})$ does not in general achieve the minimum number of regions of $\Sigma_{G}(A)$ :

Example 3.12. Consider the cycle graph $C_{4}$. As was shown in Example 3.5, we have $r\left(\Sigma_{C_{4}}(\mathrm{SHI})\right)=61$ while $r\left(\Sigma_{C_{4}}(\mathrm{SEMI})\right)=59$. 
Computing the minimum number of regions of $\Sigma_{G}(A)$ for arbitrary $G$ and finding a parameter list $A$ that achieves this minimum remain open problems. Proposition 3.4 suggests that maximizing the number of almost- $A$-admissible partial orientations may minimize the number of regions of $\Sigma_{G}(A)$, but the exact relationship between almost-admissible orientations and the number of regions remains unclear. One might wonder in particular whether the number of regions of $\Sigma_{G}(A)$ can always be minimized by a parameter list $A$ such that $a_{i j} \in\{0,1\}$ for all $\left\{v_{i}, v_{j}\right\} \in E$, as is true in the case of the complete graph.

\section{References}

B. Benson, D. Chakrabarty, and P. Tetali. G-parking functions, acyclic orientations and spanning trees. Discrete Math., 310(8):1340-1353, 2010.

A. Duval, C. Klivans, and J. Martin. The $G$-shi arrangement, and its relation to $G$-parking functions. http://www.math.utep.edu/Faculty/duval/papers/nola.pdf, January 2011.

S. Hopkins and D. Perkinson. Orientations, semiorders, arrangements, and parking functions. Elec. J. of Combin., 19(4), 2012a.

S. Hopkins and D. Perkinson. Bigraphical arrangements. eprint, arXiv:1212.4398 Forthcoming, Trans. Amer. Math. Soc., December 2012b.

A. Postnikov and B. Shapiro. Trees, parking functions, syzygies, and deformations of monomial ideals. Trans. Amer. Math. Soc., 356(8):3109-3142 (electronic), 2004.

J. Y. Shi. The Kazhdan-Lusztig cells in certain affine Weyl groups, volume 1179 of Lecture Notes in Mathematics. Springer-Verlag, Berlin, 1986.

R. P. Stanley. Hyperplane arrangements, interval orders, and trees. Proc. Nat. Acad. Sci., 93(6):26202625, 1996.

R. P. Stanley. An introduction to hyperplane arrangements. In Geometric combinatorics, volume 13 of IAS/Park City Math. Ser., pages 389-496. Amer. Math. Soc., Providence, RI, 2007. 
